\title{
SATISFACCIÓN DEL USUARIO EXTERNO CON LA ATENCIÓN EN ESTABLECIMIENTOS DEL MINISTERIO DE SALUD DE PERÚ Y SUS FACTORES ASOCIADOS
}

\author{
Akram Hernández-Vásquez ${ }^{1, a}$, Carlos Rojas-Roque ${ }^{2, b}, F$ rancisco Javier Prado-Galbarro ${ }^{3, c}$, \\ Guido Bendezu-Quispe ${ }^{4, d}$
}

\begin{abstract}
RESUMEN
Objetivos. Estimar la satisfacción con la atención en salud recibida en los establecimientos del Ministerio de Salud (MINSA) en el Perú y determinar sus factores asociados en adultos peruanos. Materiales y métodos. Estudio analítico de corte transversal empleando datos de la Encuesta Nacional de Hogares sobre Condiciones de Vida y Pobreza (ENAHO) 2018. La satisfacción con la atención en salud recibida fue calificada como: muy mala, mala, buena, y muy buena. Se describieron las características de la población en estudio mediante frecuencias y proporciones ponderadas. Para determinar los factores asociados a la satisfacción con la atención recibida, se estimó un modelo logístico ordinal generalizado. Resultados. Se incluyó un total de 14206 adultos (población expandida: 7684 602) que reportaron haber sido atendidos en algún establecimiento de salud del MINSA. A nivel nacional, el $74,3 \%$ de usuarios reportó una satisfacción con el servicio recibido como «bueno o muy bueno». Padecer una enfermedad crónica, tener una lengua materna nativa o vivir en aglomeraciones poblacionales mayores a 2000 personas se asoció con una menor satisfacción con la atención recibida. Vivir en la selva se asoció con un reporte de mayor satisfacción con la atención. Conclusiones. Tres de cada cuatro usuarios externos califican como buena o muy buena la atención recibida en establecimientos del MINSA. Existen subgrupos poblacionales con una menor satisfacción de la atención recibida. Incidir en la mejora de la satisfacción de estos usuarios mejoraría la calidad de la atención en el MINSA, el mayor prestador en salud en Perú.
\end{abstract}

Palabras clave: Encuestas y Cuestionarios; Satisfacción del Paciente; Servicios de Salud; Perú (fuente: DeCS BIREME)

\section{EXTERNAL USER SATISFACTION WITH HEALTHCARE AT FACILITIES OF THE MINISTRY OF HEALTH OF PERU AND ITS ASSOCIATED FACTORS}

\begin{abstract}
Objectives. To estimate satisfaction with the healthcare received at facilities of the Ministry of Health (MINSA) of Peru and determine its associated factors in Peruvian adults. Materials and Methods. Analytical cross-sectional study using data from the National Household Survey on Living Conditions and Poverty (ENAHO) 2018. Satisfaction with healthcare received was rated as very bad, bad, good, and very good. The characteristics of the study population were described using weighted frequencies and proportions. To determine the factors associated with satisfaction with the care received, a generalized ordinal logistic model was estimated. Results. The study included a total of 14,206 adults (expanded population: $7,684,602$ ) who reported having been treated at a MINSA healthcare facility. At the national level, $74.3 \%$ of users reported satisfaction with the service received as "good or very good". Having a chronic illness, having a native mother tongue, or living in a population with agglomerations of more than 2000 people were associated with lower satisfaction with the care received. Living in the jungle area was associated with a report of greater satisfaction with the care. Conclusions. Three out of four external users rate the care received at MINSA facilities as good or very good. There are population subgroups with less satisfaction with the care received. Improving the satisfaction of these users would improve the quality of care at the facilities of MINSA, the largest health provider in Peru.
\end{abstract}

Keywords: Surveys and Questionnaires; Patient Satisfaction; Health Services; Peru (source: MeSH NLM)

\footnotetext{
Centro de Excelencia en Investigaciones Económicas y Sociales, Vicerrectorado de Investigación, Universidad San Ignacio de Loyola. Lima, Perú. CIDS, Centro de Investigación en Demografía y Salud. León, Nicaragua.

Centro de Salud Poblacional, Instituto Nacional de Salud Pública. Cuernavaca, México.

4 Unidad de Investigación para la Generación y Síntesis de Evidencias en Salud, Vicerrectorado de Investigación, Universidad San Ignacio de Loyola. Lima, Perú.

a Médico cirujano, magíster en Gestión y Políticas Públicas; ${ }^{\mathrm{b}}$ Licenciado en Economía; ${ }^{\mathrm{c}}$ Licenciado en Estadística, doctor en Ciencias Médicas; ${ }^{\mathrm{d}}$ Médico cirujano, magíster en Informática Biomédica en Salud Global con mención en Informática en Salud.

Recibido: 04/05/2019 Aprobado: 06/11/2019 En línea: 03/12/2019
}

Citar como: Hernández-Vásquez A, Rojas-Roque C, Prado-Galbarro FJ, Bendezu-Quispe G. Satisfacción del usuario externo con la atención en establecimientos del Ministerio de Salud de Perú y sus factores asociados. Rev Peru Med Exp Salud Publica. 2019;36(4):620-8. doi: 10.17843/rpmesp.2019.364.4515. 


\section{INTRODUCCIÓN}

En las últimas décadas, existe un incremento en la evaluación de la calidad de los sistemas de salud vistos desde la perspectiva del usuario externo ${ }^{(1,2)}$, incluyendo la evaluación según el tipo de prestación de salud recibida o la evaluación del sistema de salud en general ${ }^{(2,3)}$. El estudio de los sistemas de salud desde la perspectiva de sus usuarios es útil dado que un paciente satisfecho tiene mayor adherencia al tratamiento y está dispuesto a colaborar con el proceso de atención, lo que reduciría costos innecesarios de atención ${ }^{(4,5)}$.

La Organización Mundial de la Salud (OMS) propuso en el 2000, un marco de referencia para la evaluación del desempeño de los sistemas de salud, destacando que éste no tiene únicamente la meta de mejorar y mantener la salud de la población, sino también satisfacer las necesidades de sus usuarios desde sus expectativas acerca de la atención en salud ${ }^{(6)}$. De esta forma, se resalta que la satisfacción y percepción de la calidad del servicio reportada por el usuario no depende únicamente de los resultados en salud obtenidos, sino también implica la capacidad de responder a las expectativas legítimas de la población respecto a las dimensiones no sanitarias de sus interacciones con el sistema de salud ${ }^{(6)}$.

En Perú, bajo la promulgación de la Ley № 29344 «Ley Marco del Aseguramiento Universal en Salud», se declara el derecho a la atención de salud con calidad y en forma oportuna que tiene toda la población residente en el territorio nacional ${ }^{(7)}$. El Estado Peruano busca que financiadores y prestadores de salud públicos y privados brinden un servicio de calidad sin distinción mediante un seguro de salud que le permita acceder a los distintos servicios de salud dentro del Plan Esencial de Aseguramiento en Salud (7).

A pesar de las mejoras obtenidas en aseguramiento y cobertura de atención en Perú en años recientes, se ha reportado insatisfacción por parte de los usuarios de servicios de salud. Los resultados de la Encuesta Nacional de Satisfacción de Usuarios de Salud (ENSUSALUD) del 2016, reportaron una satisfacción de los usuarios de servicios de consulta externa del $73,9 \%$ a nivel nacional (incremento de 3,8 puntos porcentuales respecto al 2014), siendo la satisfacción de los usuarios de establecimientos del Ministerio de Salud (MINSA) y Gobiernos Regionales $(66,7 \%)$ inferior al promedio nacional y al de las demás Instituciones Prestadoras de Servicios de Salud en Perú (8). Si bien, se han descrito factores asociados a la satisfacción del usuario externo en establecimientos de salud en Perú, estos estudios se corresponden a experiencias particulares, siendo necesario el estudio de estos factores a nivel nacional o de sistemas de salud ${ }^{(9,10)}$.

Teniendo en cuenta la importancia del estudio de la satisfacción de los usuarios como medida de la calidad de

\section{MENSAJES CLAVE}

Motivación para realizar el estudio. Identificar factores asociados a la satisfacción de los usuarios atención en establecimientos del Ministerio de Salud deI Perú (MINSA).

Principales hallazgos. Tres de cada cuatro usuarios externos califican como buena o muy buena la atención recibida en establecimientos del Ministerio de Salud deI Perú (MINSA). Presentar una enfermedad crónica, tener una lengua materna nativa o vivir en aglomeraciones poblacionales mayores a 2000 personas se asocia con una menor satisfacción con la atención recibida.

Implicancias. Se encontró subgrupos de usuarios que presentan menor satisfacción con la atención recibida en establecimientos del MINSA. Son necesarias estrategias que mejoren la percepción de la atención recibida por el usuario.

la atención recibida en los servicios de salud, el objetivo del estudio fue estimar la satisfacción con la atención en salud recibida en los establecimientos del Ministerio de Salud (MINSA) según departamentos del Perú y determinar sus factores asociados.

\section{MATERIALES Y MÉTODOS}

\section{DISEÑO Y POBLACIÓN DE ESTUDIO}

Se realizó un estudio analítico de corte transversal empleando datos de la Encuesta Nacional de Hogares sobre Condiciones de Vida y Pobreza (ENAHO 2018), elaborada por el Instituto Nacional de Estadística e Informática (INEI). La ENAHO es una encuesta que permite realizar estimaciones de las características sociodemográficas de la población para los niveles de inferencia nacional, urbanorural y departamental. La unidad de investigación de la encuesta está constituida por los integrantes de los hogares del país ${ }^{(11)}$.

La ENAHO recaba información sobre los siguientes temas: características de los miembros del hogar, educación, salud, empleo e ingresos, gastos del hogar y opiniones sobre gobernabilidad, democracia, discriminación, percepción de inseguridad, corrupción, entre otros. En este estudio se utilizaron datos del módulo de Educación (base enaho01a-2018-300), del módulo de Salud (base enaho01a-2018-400), del módulo Sumaria (base sumaria-2018) y del módulo de Gobernabilidad, Democracia y Transparencia (base enaho01b-2018-1).

\section{VARIABLES Y MEDICIONES}

La variable principal del estudio fue la satisfacción con la atención en salud recibida en los establecimientos del Ministerio de Salud (MINSA), medida con la siguiente 
pregunta: ¿Cómo calificaría la atención recibida en hospitales, centros de salud, postas médicas o cualquier establecimiento de salud del MINSA? Como parte de la encuesta ENAHO, esta pregunta fue consultada a los encuestados de 18 años o más que habían respondido previamente que, durante los doce meses precedentes a la encuesta, tuvieron atenciones en uno de los establecimientos de salud mencionados. Las respuestas a la pregunta sobre satisfacción con la atención recibida fue medida en escala ordinal de cuatro categorías: muy mala, mala, buena, y muy buena.

Las variables independientes del estudio fueron (en paréntesis se señalan los valores de las variables y el código alfanumérico de la pregunta registrada en el cuestionario de la ENAHO): sexo (hombre / mujer; P207_01), grupos etarios (18-29 / 30-39 / 40-49 / 50-59 / 60 o más; categorizada a partir de la pregunta $P 208 A$ ), estado civil (casado o conviviente / soltero / viudo, divorciado o separado; $P 209$ ), lengua materna aprendida durante la niñez (castellana o extranjera / nativa; $P 300 A$ ), presencia de limitaciones (sin limitaciones / con limitaciones; construida a partir de las preguntas $P 401 H 1, P 401 H 2, P 401 H 3, P 401 H 4, P 401 H 5$, y $P 401 H 6)$, quintil de bienestar económico (I (quintil más bajo) / II / III / IV / V (quintil más alto)), afiliación a un seguro de salud (sí / no; recodificado de las variables $P 4191, P 4192, P 4193$, P4194 y P4195), nivel de educación (sin nivel o nivel inicial / primaria / secundaria / superior; recodificado de la variable $P 301 A$ ), padecimiento de enfermedad crónica (sí / no; $P 401$ ), aglomeración poblacional, que son agrupaciones de personas viviendo en la misma área (mayor a 500000 habitantes / entre 100000 y 499999 habitantes / entre 2000 y 99999 habitantes / menor a 2000 habitantes; estrato) y dominio geográfico, que divide al territorio peruano según regiones naturales (Lima Metropolitana / Resto de Costa / Sierra / Selva; dominio).

Para medir el bienestar económico se consideró como variable proxy al gasto del hogar. Para ello, se dividió el gasto total anual del hogar (GASHOG2D) entre doce para obtener el gasto total mensual de la vivienda y se dividió luego entre el total de miembros del hogar (MIEPERHO) para obtener el gasto mensual per cápita del hogar. Se ordenó a los encuestados según el gasto mensual per cápita del hogar y se calcularon quintiles, estos fueron denominados quintiles de bienestar económico (el más pobre [quintil I]; el más rico [quintil V]). Las bases de datos empleadas están disponibles a libre acceso en el portal web del INEI (http://iinei.inei.gob. pe/microdatos/).

\section{ANÁLISIS ESTADÍSTICO}

Primeramente, se describieron las características sociodemográficas de la población en estudio mediante frecuencias y proporciones ponderadas incluyéndose el factor de expansión (famiegob07) y las características muestrales de la ENAHO 2018. Luego se describió la satisfacción con la atención en servicios de salud según los departamentos de Perú. Para este análisis en particular, se recodificó la variable principal de estudio en dos categorías («malo o muy malo», y «bueno o muy bueno») y se calcularon las proporciones ponderadas de los encuestados que calificaron a la atención recibida según departamentos y provincia constitucional del Callao. Los valores se representaron en un mapa utilizando el software ArcGIS Desktop (versión 10.5.1).

Las variables independientes fueron reportadas mediante frecuencias absolutas y proporciones ponderadas. Se empleó el test de chi-cuadrado para las comparaciones según las variables de interés. Previo a la estimación de los modelos de regresión, se evaluó la multicolinealidad entre las variables independientes con el objetivo de obtener parámetros precisos en las estimaciones y se consideró multicolinealidad entre las variables si el factor de inflación de la varianza superó el valor de diez. Para determinar los factores asociados a la satisfacción con la atención en salud de los adultos peruanos, se estimó un modelo logístico ordinal de probabilidades proporcionales debido al orden jerarquizado de las categorías de respuesta de la variable dependiente. Los modelos logísticos ordinales de probabilidades proporcionales han sido empleados en epidemiología y en otras aplicaciones biomédicas (12-14). Estos modelos se basan en el supuesto de probabilidades proporcionales que implica que el efecto de las probabilidades sea constante en todas las categorías de respuesta de la variable dependiente ${ }^{(15)}$. Así, el efecto de las variables independientes debe ser constante a través de todos los niveles de satisfacción.

Para probar el supuesto de líneas paralelas en el modelo estimado, se empleó la prueba de Brant ${ }^{\left({ }^{15}\right)}$. Esta prueba hace tanto una revisión global de si las variables independientes no cumplen las suposiciones de probabilidades proporcionales y una revisión de si alguna de las variables independientes no cumple por separado el supuesto. Los resultados de la prueba confirmaron que no se cumplía el supuesto de probabilidades proporcionales. Para corregir este problema, se estimó un modelo logístico ordinal generalizado, el cual relaja el supuesto y permite que el efecto de las variables independientes varíe según el punto en el que las categorías de la variable dependiente se dicotomiza. Para la inclusión de las variables independientes en el modelo logístico ordinal generalizado, éstas debieron arrojar un valor de $p<0,25$ en el análisis bivariado, punto de corte reportado en la literatura ${ }^{(16,17)}$

Para el análisis de datos se empleó el programa estadístico Stata ${ }^{\circledR}$ v14.2 (Stata Corporation, College Station, Texas, USA). Todas las pruebas estadísticas se consideraron significativas si el valor de p fue menor a 0,05 . Se reportaron odds ratio y sus respectivos intervalos de confianza al $95 \%$ (IC 95\%) de los factores incluidos en el modelo logístico ordinal.

\section{CONSIDERACIONES ÉTICAS}

La realización del estudio no requirió la aprobación de un comité de ética por tratarse de un análisis de datos 
secundarios agregados que son de dominio público y que no permite identificar a los participantes.

\section{RESULTADOS}

Se incluyeron un total de 14206 adultos (población expandida 7684 602) que reportaron haber sido atendidos en algún establecimiento de salud del MINSA durante los últimos doce meses en la ENAHO 2018 (Figura 1). La mayoría de los individuos fueron del sexo femenino $(66,7 \%)$, una quinta parte de ellos entre las edades de 30 y 39 años, y seis de cada diez de ellos eran casados o convivientes. Dos terceras partes de ellos aprendieron durante la niñez el castellano o una lengua extranjera, la gran mayoría no tiene limitaciones físicas (94,0\%), ocho de cada diez están afiliados a un seguro de salud y poco más de la mitad padece una enfermedad crónica $(54,5 \%)$ (Tabla 1$)$.

A nivel nacional, $74,3 \%$ de los individuos que fueron atendidos en algún establecimiento de salud del MINSA en los últimos doce meses, señalaron que el servicio recibido fue «bueno o muy bueno». Los departamentos que señalaron mayor porcentaje de satisfacción con el servicio de salud recibido fueron Amazonas (89,3\%), Cajamarca $(89,0 \%)$, Loreto $(87,1)$, y Huánuco $(85,1 \%)$. Por otro lado, Cusco $(56,7 \%)$, Ica $(58,4 \%)$, Tacna $(63,4 \%)$, y Madre de Dios $(65,3 \%)$ indicaron los menores porcentajes de satisfacción en el servicio de salud recibido (Figura 2).
Se evidenció asociación estadísticamente significativa entre la satisfacción con la atención recibida en establecimientos de salud del MINSA y la edad, el estado civil, lengua materna aprendida durante la niñez, presencia de limitaciones, quintil de bienestar económico, afiliación a un seguro de salud, nivel de educación, padecimiento de enfermedad crónica, aglomeración poblacional y el dominio geográfico.

Los resultados de los factores asociados a la satisfacción con la atención recibida en servicios de salud se observan en la Tabla 2. Cabe precisar que, ninguna de las variables incluidas en el análisis superó el valor de diez en el factor de inflación de la varianza. El sexo, el estado civil, las limitaciones físicas, la afiliación a un seguro de salud, el nivel de bienestar económico y el nivel de educación no se asociaron a la satisfacción con la atención percibida en los servicios de salud en Perú. Aquellas personas que habitan en la selva tienden a percibir una mayor satisfacción en los servicios de salud en comparación a quienes habitan en la región costa. Sin embargo, este efecto se pierde cuando las personas de la selva señalan la satisfacción con la atención como muy mala. Por otro lado, las personas que habitan en aglomeraciones poblacionales mayores a 2000 habitantes perciben una peor satisfacción con la atención recibida en comparación a quienes viven en aglomeraciones menores a 2000 habitantes (correspondientes a áreas rurales). Asimismo, quienes tienen una enfermedad crónica o una lengua materna nativa aprendida durante la niñez

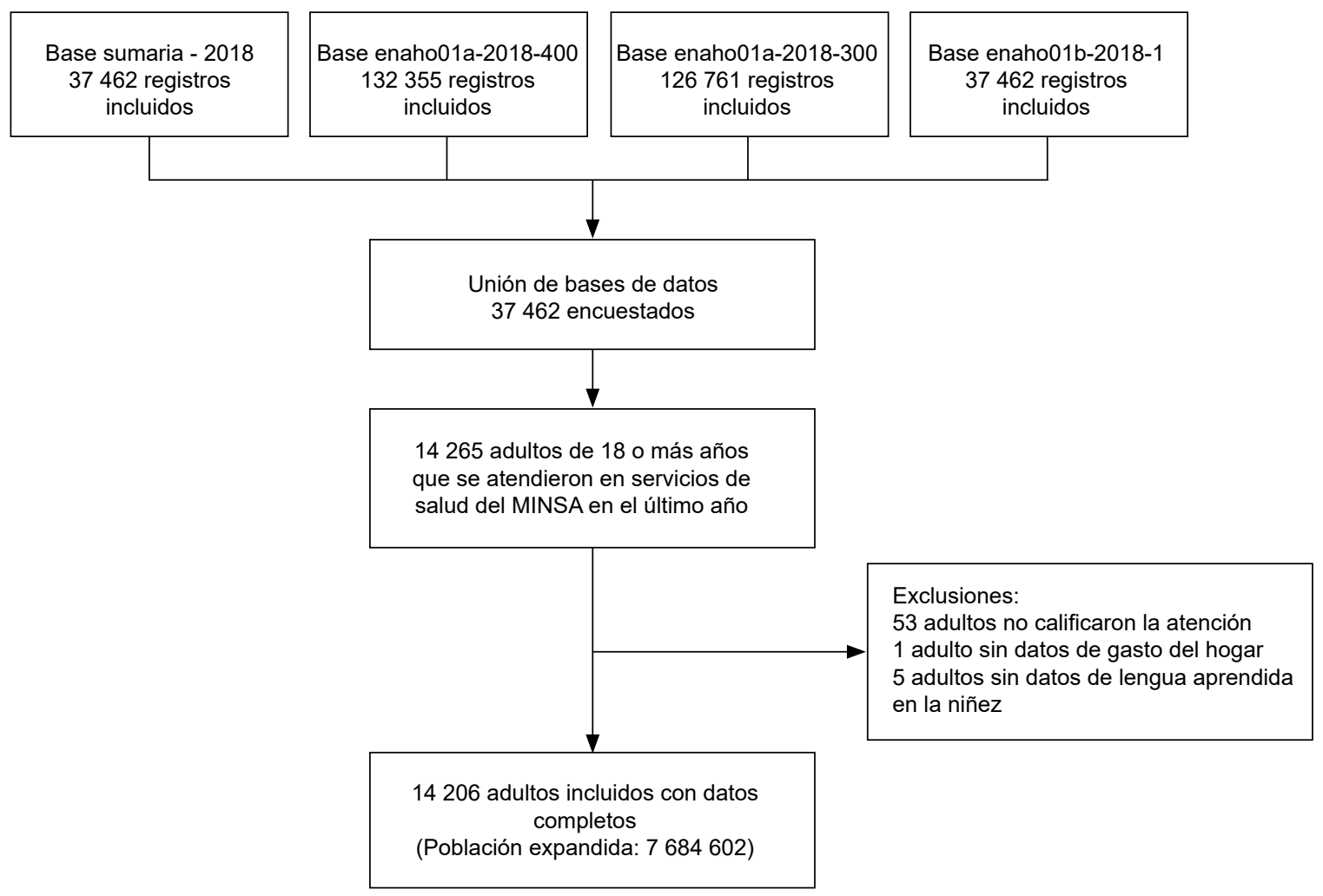

Figura 1. Flujograma de participantes incluidos en el estudio 
Tabla 1. Características y calificación de la atención recibida de los usuarios que acudieron a establecimientos del Ministerio de Salud del Perú, ENAHO 2018

\begin{tabular}{|c|c|c|c|c|c|c|c|}
\hline \multirow{3}{*}{ Características } & \multirow{2}{*}{\multicolumn{2}{|c|}{$\begin{array}{l}\text { Adultos encuestados } \\
\qquad(n=14206)\end{array}$}} & \multicolumn{4}{|c|}{ Calificación de la atención recibida } & \multirow[b]{3}{*}{ Valor de $\mathrm{p}^{*}$} \\
\hline & & & \multirow{2}{*}{$\begin{array}{c}\text { Muy mala } \\
\%\end{array}$} & \multirow{2}{*}{$\begin{array}{c}\text { Mala } \\
\%\end{array}$} & \multirow{2}{*}{$\begin{array}{c}\text { Buena } \\
\%\end{array}$} & \multirow{2}{*}{$\begin{array}{c}\begin{array}{c}\text { Muy } \\
\text { buena }\end{array} \\
\% \\
\end{array}$} & \\
\hline & Frecuencia & $\%$ & & & & & \\
\hline \multicolumn{8}{|l|}{ Sexo } \\
\hline Hombre & 4928 & 33,3 & 4,5 & 20,7 & 71,9 & 2,9 & 0,528 \\
\hline Mujer & 9278 & 66,7 & 3,5 & 22,4 & 71,5 & 2,6 & \\
\hline \multicolumn{8}{|l|}{ Edades (años) } \\
\hline $18-29$ & 2413 & 24,0 & 4,1 & 20,2 & 71,8 & 3,8 & 0,087 \\
\hline $30-39$ & 2849 & 19,3 & 3,6 & 21,9 & 71,8 & 2,7 & \\
\hline $40-49$ & 2641 & 18,3 & 3,7 & 26,0 & 68,5 & 1,8 & \\
\hline $50-59$ & 2209 & 15,4 & 4,3 & 24,9 & 69,2 & 1,6 & \\
\hline 60 o más & 4094 & 23,0 & 3,5 & 18,2 & 75,3 & 3,0 & \\
\hline \multicolumn{8}{|l|}{ Estado civil } \\
\hline Casado o conviviente & 8688 & 63,3 & 3,2 & 21,9 & 72,7 & 2,2 & 0,107 \\
\hline Soltero & 1729 & 16,3 & 5,9 & 21,1 & 70,3 & 2,7 & \\
\hline Viudo, divorciado o separado & 3789 & 20,4 & 4,1 & 22,4 & 69,3 & 4,3 & \\
\hline \multicolumn{8}{|c|}{ Lengua materna aprendida durante la niñez } \\
\hline Castellano o lengua extranjera & 8238 & 67,9 & 4,0 & 22,2 & 70,7 & 3,1 & 0,204 \\
\hline Nativa & 5968 & 32,1 & 3,4 & 21,0 & 73,6 & 1,9 & \\
\hline \multicolumn{8}{|l|}{ Presencia de limitaciones } \\
\hline Sin limitaciones & 13005 & 94,0 & 3,7 & 22,4 & 71,2 & 2,8 & 0,024 \\
\hline Con limitaciones & 1201 & 6,0 & 6,3 & 13,7 & 78,6 & 1,4 & \\
\hline \multicolumn{8}{|l|}{ Quintil de bienestar económico } \\
\hline I (más bajo) & 5928 & 32,5 & 2,9 & 14,7 & 80,6 & 1,8 & $<0,001$ \\
\hline II & 3602 & 26,3 & 4,3 & 22,1 & 71,8 & 1,8 & \\
\hline III & 2271 & 19,1 & 4,6 & 24,8 & 66,4 & 4,2 & \\
\hline IV & 1536 & 14,1 & 3,0 & 32,9 & 59,8 & 4,2 & \\
\hline V (más alto) & 869 & 8,0 & 5,5 & 23,6 & 67,7 & 3,2 & \\
\hline \multicolumn{8}{|l|}{ Afiliación a un seguro de salud } \\
\hline Sí & 12351 & 83,4 & 3,8 & 20,6 & 73,0 & 2,7 & 0,022 \\
\hline No & 1855 & 16,6 & 4,0 & 28,4 & 64,7 & 2,9 & \\
\hline \multicolumn{8}{|l|}{ Nivel de educación } \\
\hline Sin nivel o nivel inicial & 1907 & 9,1 & 2,8 & 17,5 & 76,4 & 3,3 & 0,004 \\
\hline Primaria & 5907 & 35,1 & 2,7 & 18,3 & 76,7 & 2,2 & \\
\hline Secundaria & 4475 & 38,3 & 5,1 & 24,0 & 68,0 & 2,9 & \\
\hline Superior & 1917 & 17,5 & 3,9 & 26,5 & 66,6 & 2,9 & \\
\hline \multicolumn{8}{|l|}{ Padecimiento de enfermedad crónica } \\
\hline Sí & 7956 & 54,5 & 4,6 & 23,8 & 69,2 & 2,5 & 0,004 \\
\hline No & 6250 & 45,5 & 2,9 & 19,5 & 74,5 & 3,0 & \\
\hline \multicolumn{8}{|l|}{ Aglomeración poblacional (habitantes) } \\
\hline Mayor a 500000 & 542 & 34,8 & 5,9 & 29,4 & 60,6 & 4,2 & $<0,001$ \\
\hline Entre 100000 y 499999 & 1659 & 28,2 & 3,9 & 26,9 & 65,9 & 3,2 & \\
\hline Entre 2000 y 99999 & 3485 & 12,7 & 3,7 & 22,4 & 72,0 & 1,9 & \\
\hline Menor a 2000 & 8520 & 24,3 & 2,5 & 14,3 & 81,0 & 2,1 & \\
\hline \multicolumn{8}{|l|}{ Dominio geográfico } \\
\hline Costa & 3040 & 41,0 & 5,0 & 28,2 & 63,1 & 3,7 & $<0,001$ \\
\hline Sierra & 7775 & 43,5 & 3,2 & 18,3 & 76,8 & 1,8 & \\
\hline Selva & 3391 & 15,5 & 2,5 & 15,1 & 79,8 & 2,6 & \\
\hline
\end{tabular}

* Significancia estadística obtenida mediante la prueba de chi-cuadrado entre las categorías de la calificación de la atención recibida y las covariables de estudio.

\%: Proporción ponderada que incluyó el factor de expansión y especificaciones muestrales de la ENAHO 2018

ENAHO: Encuesta Nacional de Hogares sobre Condiciones de Vida y Pobreza. 


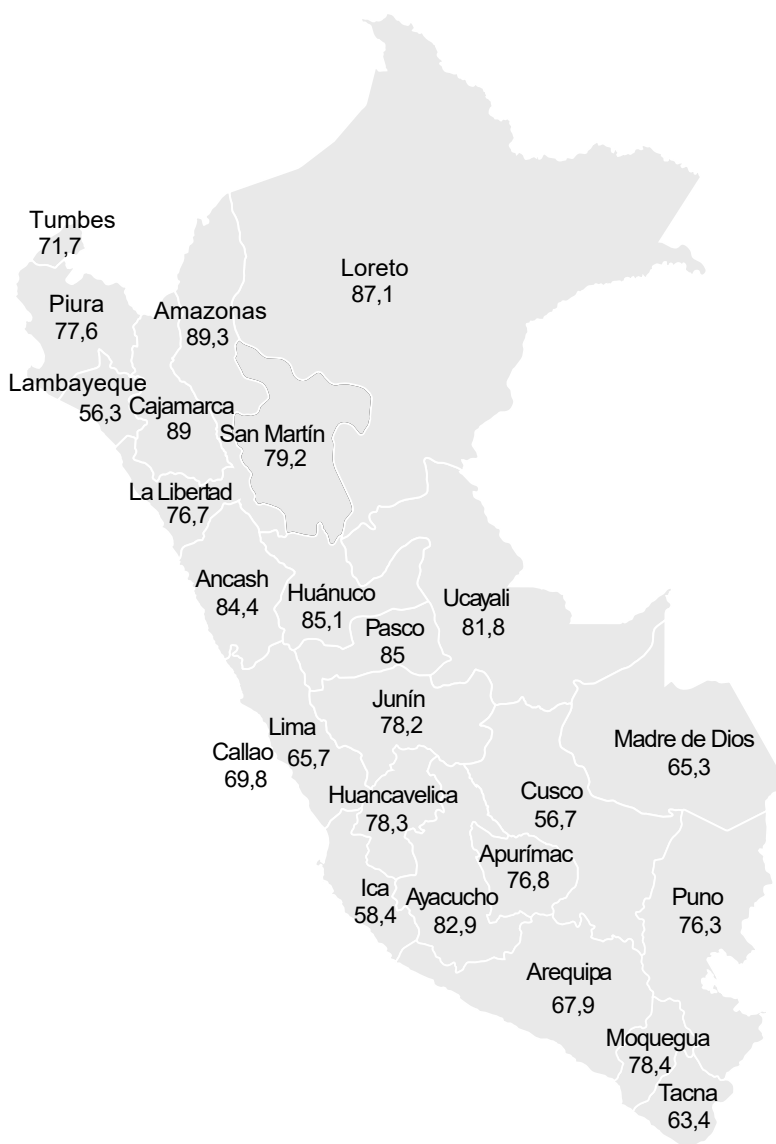

Figura 2. Porcentaje de usuarios según departamentos que califica como «buena o muy buena» la atención recibida en establecimientos de salud del Ministerio de Salud del Perú, ENAHO 2018

perciben una peor satisfacción con la atención en salud en comparación a quienes no tienen enfermedades crónicas o aprendieron durante la niñez el castellano u otra lengua extranjera como lengua materna. Se encontró que las personas entre 40 y 59 años perciben una peor satisfacción con la atención en salud en comparación a las personas que tienen entre 18 y 29 años. Los adultos mayores no tuvieron diferencia en comparación a personas entre 18 y 29 años en cuanto a la satisfacción percibida durante la atención en salud.

\section{DISCUSIÓN}

Se buscó estimar y determinar los factores asociados a la satisfacción con la atención recibida por los adultos que acudieron a establecimientos de salud del MINSA de Perú. Se encontró que casi tres cuartas partes de la población atendida en establecimientos del MINSA calificaron como buena o muy buena la atención recibida. Las personas que padecen una enfermedad crónica, que aprendieron durante su niñez una lengua nativa como lengua materna y los que viven en aglomeraciones poblacionales mayores a 2000 personas presentaron una menor satisfacción con la atención recibida en establecimientos de salud del MINSA.
La proporción de usuarios que calificó la atención recibida como buena o muy buena fue similar al reportado por la encuesta ENSUSALUD del 2016 en cuanto a la satisfacción de los usuarios atendidos a nivel nacional por consultorio externo $(73,9 \%)$ y ligeramente mayor al reporte de satisfacción de usuarios externos del MINSA y establecimientos de Gobiernos Regionales respecto a atenciones que ocurrieron durante el $2016(66,7 \%){ }^{(8)}$, ambos valores superiores a los reportados para el $200{ }^{(10)}$. Un estudio realizado en un hospital infantil de referencia nacional encontró que los usuarios reportaron satisfacción respecto a la atención en consulta ambulatoria, sin embargo, reportó insatisfacción respecto a la higiene en el establecimiento ${ }^{(9)}$. La satisfacción del usuario con la atención recibida no depende únicamente de los resultados de salud obtenidos. Por ello, es necesario que el sistema de salud tenga en cuenta las expectativas de sus usuarios al haberse para obtener los beneficios descritos en literatura sobre el usuario satisfecho tales como un mejor cumplimiento del tratamiento y una reducción de costos para el sistema de salud.

En general, los usuarios de los departamentos de la costa presentaron menor satisfacción con la atención recibida en establecimientos del MINSA. Este resultado se corresponde con una menor satisfacción con la atención percibida en aglomeraciones poblacionales de mayor tamaño, explicable considerando que la costa contiene a las capitales y ciudades de mayor tamaño poblacional. Un hallazgo del estudio fue que las aglomeraciones poblaciones más pequeñas tendrían una mayor satisfacción. En Perú, las zonas rurales suelen representar las aglomeraciones poblacionales más pequeñas y se presentan con más frecuencia en los dominios geográficos de la sierra y selva del país, misma situación para la población que presenta aprendizaje de una lengua nativa como lengua materna durante la niñez. Se ha reportado que usuarios de servicios de salud en zonas rurales (zonas de baja aglomeración poblacional) reportan una buena satisfacción en la atención a pesar de considerar la posibilidad de mejoras en la misma como respeto a sus costumbres, la disponibilidad de insumos para su atención y los tiempos para la atención ${ }^{(18,19)}$. Los factores previamente descritos actuarían como barreras que limitan el acceso a personas de comunidades nativas en su acceso y confort para el uso de servicios de salud ${ }^{(20)}$, por lo que es prioritario la implementación de estrategias con adecuación cultural que complementan a una atención de calidad en salud más allá del componente técnico de la misma.

Las personas con una enfermedad crónica reportaron una menor satisfacción sobre la atención recibida. Al respecto, personas con enfermedades no transmisibles o que tienen una percepción de un mal estado de salud son más propensos a reportar una peor satisfacción de la atención en los servicios de salud (21), lo que podría explicar este hallazgo. Por otro lado, en relación a la edad, se halló 
Tabla 2. Factores asociados a la satisfacción con la atención en establecimientos del Ministerio de Salud del Perú, ENAHO 2018

\begin{tabular}{|c|c|c|c|c|c|c|}
\hline \multirow{2}{*}{ Características } & \multicolumn{2}{|c|}{$\begin{array}{l}\text { Malo, Bueno o Muy bueno } \\
\text { vs. Muy malo }\end{array}$} & \multicolumn{2}{|c|}{$\begin{array}{l}\text { Bueno o Muy bueno vs. } \\
\text { Muy malo y malo }\end{array}$} & \multicolumn{2}{|c|}{$\begin{array}{l}\text { Muy Bueno vs Muy malo, } \\
\text { Malo o Bueno }\end{array}$} \\
\hline & OR (IC 95\%) & $\begin{array}{l}\text { Valor } \\
\text { de } p\end{array}$ & OR (IC 95\%) & $\begin{array}{l}\text { Valor } \\
\text { de } p\end{array}$ & OR (IC 95\%) & $\begin{array}{l}\text { Valor } \\
\text { de } p\end{array}$ \\
\hline \multicolumn{7}{|l|}{ Edades (años) } \\
\hline $18-29$ & Referencia & & Referencia & & Referencia & \\
\hline $30-39$ & $0,84(0,62-1,15)$ & 0,279 & $0,84(0,62-1,15)$ & 0,279 & $0,84(0,62-1,15)$ & 0,279 \\
\hline $40-49$ & $0,67(0,49-0,90)$ & 0,009 & $0,67(0,49-0,90)$ & 0,009 & $0,67(0,49-0,90)$ & 0,009 \\
\hline $50-59$ & $0,70(0,51-0,97)$ & 0,031 & $0,70(0,51-0,97)$ & 0,031 & $0,70(0,51-0,97)$ & 0,031 \\
\hline 60 o más & $0,96(0,67-1,37)$ & 0,809 & $0,96(0,67-1,37)$ & 0,809 & $0,96(0,67-1,37)$ & 0,809 \\
\hline \multicolumn{7}{|l|}{ Estado civil } \\
\hline Casado o conviviente & Referencia & & Referencia & & Referencia & \\
\hline Soltero & $0,85(0,58-1,24)$ & 0,393 & $0,85(0,58-1,24)$ & 0,393 & $0,85(0,58-1,24)$ & 0,393 \\
\hline Viudo, divorciado o separado & $0,93(0,73-1,18)$ & 0,550 & $0,93(0,73-1,18)$ & 0,550 & $0,93(0,73-1,18)$ & 0,550 \\
\hline \multicolumn{7}{|l|}{$\begin{array}{l}\text { Lengua materna aprendida } \\
\text { durante la niñez }\end{array}$} \\
\hline Castellano o lengua extranjera & Referencia & & Referencia & & Referencia & \\
\hline Nativa & $0,78(0,64-0,95)$ & 0,012 & $0,78(0,64-0,95)$ & 0,012 & $0,78(0,64-0,95)$ & 0,012 \\
\hline \multicolumn{7}{|l|}{ Presencia de limitaciones } \\
\hline Sin limitaciones & Referencia & & Referencia & & Referencia & \\
\hline Con limitaciones & $1,18(0,79-1,75)$ & 0,427 & $1,18(0,79-1,75)$ & 0,427 & $1,18(0,79-1,75)$ & 0,427 \\
\hline \multicolumn{7}{|l|}{ Quintil de bienestar económico } \\
\hline I (más bajo) & Referencia & & Referencia & & Referencia & \\
\hline II & $0,92(0,53-1,61)$ & 0,777 & $0,79(0,65-0,96)$ & 0,019 & $1,05(0,69-1,58)$ & 0,830 \\
\hline III & $1,00(0,58-1,74)$ & 0,988 & $0,81(0,63-1,05)$ & 0,110 & $2,58(1,43-4,67)$ & 0,002 \\
\hline IV & $1,74(0,83-3,66)$ & 0,146 & $0,66(0,49-0,89)$ & 0,007 & $2,54(1,11-5,82)$ & 0,028 \\
\hline V (más alto) & $0,95(0,39-2,30)$ & 0,913 & $0,99(0,66-1,49)$ & 0,974 & $2,11(0,91-4,85)$ & 0,080 \\
\hline \multicolumn{7}{|l|}{ Afiliación a un seguro de salud } \\
\hline No & Referencia & & Referencia & & Referencia & \\
\hline Sí & $1,14(0,89-1,46)$ & 0,305 & $1,14(0,89-1,46)$ & 0,305 & $1.14(0,89-1,46)$ & 0,305 \\
\hline \multicolumn{7}{|l|}{ Nivel de educación } \\
\hline Sin nivel o nivel inicial & Referencia & & Referencia & & Referencia & \\
\hline Primaria & $1,14(0,67-1,94)$ & 0,637 & $1,07(0,82-1,39)$ & 0,614 & $0,61(0,16-2,29)$ & 0,468 \\
\hline Secundaria & $0,64(0,40-1,04)$ & 0,070 & $0,83(0,62-1,10)$ & 0,196 & $0,61(0,14-2,56)$ & 0,496 \\
\hline Superior & $0,81(0,45-1,46)$ & 0,490 & $0,79(0,55-1,14)$ & 0,215 & $0,51(0,11-2,43)$ & 0,399 \\
\hline \multicolumn{7}{|l|}{ Padecimiento de enfermedad crónica } \\
\hline No & Referencia & & Referencia & & Referencia & \\
\hline Sí & $0,72(0,60-0,88)$ & 0,001 & $0,72(0,60-0,88)$ & 0,001 & $0,72(0,60-0,88)$ & 0,001 \\
\hline \multicolumn{7}{|l|}{ Aglomeración poblacional (habitantes) } \\
\hline Menor a 2000 & Referencia & & Referencia & & Referencia & \\
\hline Entre 2000 y 99999 & $0,69(0,59-0,80)$ & $<0,001$ & $0,69(0,59-0,80)$ & $<0,001$ & $0,69(0,59-0,80)$ & $<0,001$ \\
\hline Entre 100000 y 499999 & $0,60(0,49-0,74)$ & $<0,001$ & $0,60(0,49-0,74)$ & $<0,001$ & $0,60(0,49-0,74)$ & $<0,001$ \\
\hline Mayor a 500000 & $0,60(0,44-0,82)$ & 0,001 & $0,60(0,44-0,82)$ & 0,001 & $0,60(0,44-0,82)$ & 0,001 \\
\hline \multicolumn{7}{|l|}{ Dominio geográfico } \\
\hline Costa & Referencia & & Referencia & & Referencia & \\
\hline Sierra & $1,30(0,82-2,08)$ & 0,263 & $1,32(1,09-1,60)$ & 0,005 & $0,53(0,31-0,89)$ & 0,016 \\
\hline Selva & $1,65(1,02-2,67)$ & 0,040 & $1,71(1,40-2,10)$ & $<0,001$ & $0,74(0,44-1,22)$ & 0,237 \\
\hline
\end{tabular}

Las estimaciones incluyeron el factor de expansión y especificaciones muestrales de la ENAHO 2018.

ENAHO: Encuesta Nacional de Hogares sobre Condiciones de Vida y Pobreza; OR: Odds ratio. 
que los grupos etarios mayores a los adultos jóvenes presentaron una mayor satisfacción con la atención recibida, aunque no existió diferencia entre los adultos más jóvenes y los adultos mayores. Se ha reportado que personas de grupos etarios mayores presentan una mayor satisfacción en el uso de servicios de salud, aunque esta satisfacción presentaría una disminución en los grupos etarios de mayor edad, habiéndose señalado como posible explicación para este fenómeno el que los grupos etarios mayores pondrían una mayor valoración las habilidades técnicas del personal médico respecto a habilidades interpersonales ${ }^{(22-24)}$.

Sobre la percepción negativa de la satisfacción con la atención de subgrupos de usuarios del MINSA, se puede señalar que, si bien la mayor parte de la población atendida en establecimientos de salud del MINSA y de los Gobiernos regionales $(89,6 \%)$ cuenta con algún tipo de seguro, y que esta disposición se encuentra asociada a una mayor satisfacción respecto a la atención, múltiples factores relacionados a insatisfacción de los usuarios con la atención médica están presentes en los usuarios del MINSA. Para el 2016, el tiempo de espera promedio para la atención en consultorio externo para usuarios de servicios de salud del MINSA y de hospitales de los Gobiernos Regionales fue en promedio de 135 minutos, 34 minutos más que la media nacional, siendo además este promedio mayor al de otros sistemas de aseguramiento en salud del país ${ }^{(8)}$. Adicional a ello, el tiempo de atención promedio fue de 12 minutos, valor inferior al reportado en el sector privado ${ }^{(8)}$. Además, únicamente el $63,1 \%$ de la población que acudió a establecimientos del MINSA y de los Gobiernos Regionales obtuvo una cita o turno de atención por consulta externa el mismo día de la atención. Otro motivo que podría relacionarse es que, a pesar que el $89,7 \%$ de establecimientos del MINSA y gobiernos regionales se encuentran en la misma localidad del usuario, en caso de que la atención de salud requiere movilizarse a otra ciudad, la mitad de los usuarios va directamente desde su hogar a la atención debido a los costos que puede generar la movilización y el alojamiento en una zona diferente ${ }^{(8)}$

Dentro de las limitaciones del presente estudio, se tiene que los datos sobre las respuestas obtenidas durante las entrevistas de la encuesta ENAHO para evaluar la satisfacción de los usuarios puede verse influenciada por la temporalidad, la manera en que éstas son consultadas y errores de registro. Si bien la perspectiva del usuario es importante para determinar la calidad de la atención brindada en los establecimientos de salud, se debe mencionar que la relación entre la satisfacción del paciente y los resultados y la atención de salud recibida puede no ser consistente ${ }^{(25,26)}$, pues haber recibido una atención de calidad puede no reflejar un puntaje de satisfacción positiva de la atención por el usuario y viceversa (27); de igual forma, se ha visto que haber recibido un servicio discrecional aumenta la satisfacción reportada por el usuario a pesar que éste no mejora o la mejora que pueda ofrecer a la condición de salud del paciente es mínima ${ }^{(28)}$. Adicionalmente, dado que los datos en la encuesta ENAHO no consignan el nivel de atención del establecimiento de salud evaluado, no fue posible hacer una distinción entre la satisfacción de los usuarios en cuanto a la atención recibida según niveles de atención, lo que podría ayudar a entender mejor el fenómeno estudiado. A pesar de ello, consideramos que el modelo presentado incluye factores previamente reportados y asociados a la satisfacción con la atención recibida por los usuarios en los sistemas de salud, obtenidos de una encuesta con representatividad nacional que cuenta con un sistema de control de calidad de los datos recolectados, lo que hace a los resultados útiles para tener una visión general acerca del objeto de estudio.

Concluimos que, el vivir en aglomeraciones poblacionales de tamaño mayor, como las ciudades, padecer una enfermedad crónica y tener una lengua nativa como lengua materna se asocia a una menor satisfacción con la atención recibida en establecimientos de salud del MINSA. Por otro lado, residir en la región selva se asoció a una mayor satisfacción en cuanto a la atención recibida. Es necesario profundizar en el estudio de factores que condicionan una baja satisfacción con la atención recibida en población que acude a establecimientos de salud del MINSA, teniendo presente que este sistema de salud agrupa a la mayor proporción de población peruana afiliada a un seguro y tiene la mayor oferta de servicios de salud en Perú.

Contribuciones de autoría: AHV tuvo la idea de investigación, diseñó el estudio, recopiló y procesó los datos. AHV y CRR analizaron los datos bajo la supervisión de FJPG. Todos los autores participaron en la interpretación de los datos, redacción del manuscrito y aprobaron la versión final.

Fuentes de financiamiento: autofinanciado.

Conflictos de interés: AHV es miembro del comité editor de la RPMESP y no tuvo participación en ninguna etapa del proceso editorial posterior al envío del presente artículo, asumiendo únicamente el rol de autor. Los demás autores declaran no tener conflictos de interés.

\section{REFERENCIAS BIBLIOGRÁFICAS}

1. Crow H, Gage H, Hampson S, Hart J, Kimber A, Storey L, et al. Measurement of satisfaction with health care: Implications for practice from a systematic review of the literature. Health technology assessment. Health Technol Assess. 2002;6(32):1-244.

2. Sofaer $S$, Firminger K. Patient perceptions of the quality of health services. Annu Rev
Public Health. 2005;26:513-59.

3. Jackson JL, Chamberlin J, Kroenke K. Predictors of patient satisfaction. Soc Sci Med. 2001;52(4):609-20. 
4. Blendon RJ, Schoen C, DesRoches C, Osborn R, Zapert K. Common concerns amid diverse systems: health care experiences in five countries. Health Aff (Millwood). 2003;22(3):106-21.

5. Blendon RJ, Leitman R, Morrison I, Donelan K. Satisfaction with health systems in ten nations. Health Aff (Millwood). 1990;9(2):185-92.

6. World Health Organization. The World Health Report 2000. Health Systems: Improving Performance [Internet]. Geneva: WHO; 2018 [citado el 30 abril de 2019]. Disponible en: https://www.who.int/ whr/2000/en/whr00_en.pdf?ua=1.

7. Ministerio de Salud. Aseguramiento Universal en Salud - AUS [Internet]. Lima: MINSA; 2010 [citado el 25 abril de 2019]. http://www.sis.gob.pe/Portal/ productos/aus/index.html.

8. Instituto Nacional de Estadística e Informática. Encuesta Nacional de Satisfacción de Usuarios en Salud 2016 [Internet]. Lima: INEI; 2017 [citado el 20 marzo de 2019]. Disponible en: http://portal.susalud.gob.pe/wpcontent/uploads/archivo/encuestasat-nac/2016/INFORME_FINAL_ ENSUSALUD_2016.pdf.

9. Shimabuku R, Huicho L, Fernández D, Nakachi G, Maldonado R, Barrientos A. Niveles de insatisfacción del usuario externo en el Instituto Nacional de Salud del Niño de Lima, Perú. Rev Peru Med Exp Salud Publica. 2012;29(4):483-9.

10. Seclén-Palacin J, Darras C, editors. Satisfacción de usuarios de los servicios de salud: factores sociodemográficos y de accesibilidad asociados: Perú, 2000. An Fac Med. 2005;66(2): 127-141.

11. Instituto Nacional de Estadística e Informática. Encuesta Nacional de Hogares [Internet]. Lima: INEI;2019 [citado el 15 abril de 2019]. Disponible en: https://webinei.inei.gob.pe/anda_inei/ index.php/catalog/613.

12. Lee J. Cumulative logit modelling for ordinal response variables: applications to biomedical research. Comput Appl Biosci. 1992;8(6):555-62

13. Scott SC, Goldberg MS, Mayo NE. Statistical assessment of ordinal outcomes in comparative studies. J Clin Epidemiol. 1997;50(1):45-55

14. Walters SJ, Campbell MJ, Lall R. Design and analysis of trials with quality of life as an outcome: a practical guide. J Biopharm Stat. 2001;11(3):155-76

15. Liu X. Applied ordinal logistic regression using Stata: From single-level to multilevel modeling. 1ra ed. Londres: Sage Publications; 2015.

16. Bendal RB, Afifi AA. Comparison of stopping rules in forward regression. J Am Stat Assoc. 1977;72(357):46-53.

17. Mickey RM, Greenland S. The impact of confounder selection criteria on effect estimation. Am J Epidemiol. 1989;130(5):125-37.

18. Ganguly E, Sharma PK. Client Satisfaction with Quality of Health Care in a Rural area in Southern India. J Public Health Epidemiol. 2014;6(8):239-45.

19. Kuzma J, Solomom J, Masono A, Manari B, Hopping J, Pasum E, et al. Assessment of patients' satisfaction with rural health services. Contemporary PNG Studies. 2012;17:60

20. Defensoría del Pueblo. La Salud de las Comunidades Nativas: Un reto para el Estado [Internet]. Lima: Defensoría del Pueblo; 2008 [citado el 20 abril de 2019]. Disponible en: http://www.servindi.org/ pdf/DP_informe_134.pdf.

21. Bleich SN, Özaltin E, Murray CJ. How does satisfaction with the health-care system relate to patient experience. Bul World Health Organ. 2009;87(4):271-8.
22. Jaipaul CK, Rosenthal GE. Are older patients more satisfied with hospital care than younger patients. J Gen Intern Med. 2003;18(1):23-30.

23. Lee Y, Kasper JD. Assessment of medical care by elderly people: general satisfaction and physician quality. Health Serv Res. 1998;32(6):741-58.

24. Nguyen Thi PL, Briancon S, Empereur F, Guillemin F. Factors determining inpatient satisfaction with care. Soc Sci Med. 2002;54(4):493-504.

25. Rao JK, Weinberger M, Kroenke K. Visitspecific expectations and patient-centered outcomes: a literature review. Arch Fam Med. 2000;9(10):1148.

26. Sequist TD, Schneider EC, Anastario M, Odigie EG, Marshall R, Rogers WH, et al. Quality monitoring of physicians: linking patients' experiences of care to clinical quality and outcomes. J Gen Intern Med. 2008;23(11):1784-90.

27. Anhang Price R, Elliott MN, Zaslavsky AM, Hays RD, Lehrman WG, Rybowski $\mathrm{L}$, et al. Examining the role of patient experience surveys in measuring health care quality. Med Care Res Rev 2014;71(5):522-54.

28. Kravitz RL, Epstein RM, Feldman MD, Franz CE, Azari R, Wilkes MS, et al. Influence of patients' requests for directto-consumer advertised antidepressants: a randomized controlled trial. Jama. 2005;293(16):1995-2002.

Correspondencia: Akram Abdul Hernández Vásquez

Dirección: Universidad San Ignacio de Loyola, Av. La Fontana 550, La Molina, Lima, Perú

Teléfono: (00511) 3851492

Correo electrónico: ahernandez@usil.edu.pe 